\title{
Impact of dietary practices on metabolic health in Omani adults
}

\author{
K. Al Zuhaibi, F. McCullough and A.M. Salter \\ University of Nottingham, Loughborough, Leicestershire, LE12 5RD, UK
}

The Sultanate of Oman, like many Arab Gulf countries, has experienced rapid changes in diet and lifestyle as a result of globalization, urbanization and economic growth. In 2014, the World Health Organization (WHO) estimated that non-communicable diseases (NCDs) accounted for $68 \%$ of deaths in Oman ${ }^{1}$. Cardiovascular disease, diabetes and other NCDs accounted for $33 \%, 10 \%$ and $13 \%$ of deaths respectively. Obesity, a risk factor many NCDs, has been estimated to affect $19.4 \%$ of male and $25.9 \%$ of female adults, aged 20 years and above, in Oman ${ }^{2}$. In spite of the prevalence of chronic diseases, there is a dearth of information about the current dietary practices and physical activity among Omani adults. The present study aimed to; assess food choices and dietary intake of Omani adults across all Governorates and to explore the prominent sources of energy in the diet. In order to assess these variables, a survey study was conducted on 500 healthy adults, aged (18-60 years), living in Oman during the period from December 2013 to April 2014. The study was approved by the University of Nottingham Medical School Ethics Committee and a questionnaire was designed and piloted to ensure it was culturally appropriate to the study population. Trained health educators from all Omani Governorates were recruited to administrate and distribute the questionnaires. Eligible participants completed questionnaires, specifically designed for this study, which included demographic data and 3-day dietary assessment. Collected data were statistically analysed by ANOVA.

\begin{tabular}{|c|c|c|c|c|}
\hline & \multicolumn{2}{|l|}{ Male } & \multicolumn{2}{|l|}{ Female } \\
\hline & $18-30 y$ & $31-60 y$ & $18-30 y$ & $31-60 y$ \\
\hline BMI $\left(\mathrm{kg} / \mathrm{m}^{2}\right)$ & $26 \pm 5$ & $28 \pm 6$ & $23 \pm 5$ & $28 \pm 6$ \\
\hline Total energy $(\mathrm{kcal} / \mathrm{d})$ & $2353 \pm 592$ & $2360 \pm 549$ & $2145 \pm 516$ & $2188 \pm 547$ \\
\hline$\%$ Energy $\mathrm{CHO}$ & $49 \cdot 4 \pm 7 \cdot 6$ & $52 \pm 6 \cdot 8$ & $50 \cdot 8 \pm 6 \cdot 3$ & $50 \cdot 9 \pm 6 \cdot 3$ \\
\hline$\%$ Energy Protein & $16 \cdot 3 \pm 3 \cdot 5$ & $16 \cdot 4 \pm 2 \cdot 9$ & $15 \cdot 7 \pm 3 \cdot 1$ & $15 \cdot 2 \pm 2 \cdot 8$ \\
\hline$\%$ Energy from Fat & $32 \cdot 9 \pm 7 \cdot 1$ & $29 \cdot 9 \pm 6 \cdot 6$ & $32 \cdot 1 \pm 6 \cdot 6$ & $32 \cdot 4 \pm 6 \cdot 5$ \\
\hline
\end{tabular}

Values are means \pm Standard deviation (SD)

Results showed no significant effects of governorates (Urban $v$ s Rural) on BMI of study participants. By contrast, BMI was significantly $(p<0.05)$ affected by gender and age group. Although males showed more excessive weight than females in general, BMI was similar between males and females in the older age group. Total energy intake was higher in males than females $(p<0 \cdot 05)$ though no significant effect of age was found. The distribution of energy intake between the major macronutrients was similar to that seen in many Western countries. Proportion of energy from protein was statistically $(p<0 \cdot 05)$ different between genders. The moderate daily mean energy intakes of the study samples compared to their BMI suggest low levels of energy expenditure, rather than intake, may represent a significant problem amongst Omani adults. To conclude, the prevalence of overweight amongst adults, particularly those over 30y, found in this study, support the view of adopting preventative and intervention strategies, targeting unhealthy eating behaviours and physical inactivity, in order to reduce high prevalence of diet-related chronic disease in Oman.

K.A-Z was supported by a PhD studentship provided by the Omani government

1. World Health Organization (2014). Noncommuniocable Diseases (NCD) Country Profiles. Geneva: WHO.

2. World Health Organization (2008). Oman Statistical Profile. Geneva: WHO. 